\title{
Molecular modelling studies on 2-amino 6-aryl-sulphonylbenzonitriles as non-nucleoside reverse transcriptase inhibitors of HIV-1: A QSPR approach
}

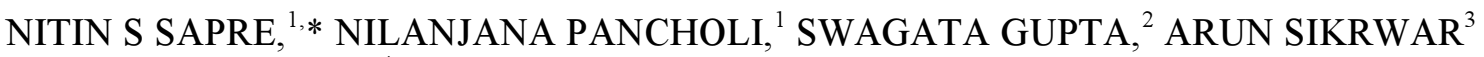 \\ and NEELIMA SAPRE ${ }^{4}$ \\ ${ }^{1}$ Department of Applied Chemistry, SGSITS, Indore 452001 \\ ${ }^{2}$ Department of Chemistry, Government P.G. College, MHOW \\ ${ }^{3}$ Department of Chemistry, Government Holkar Science College, Indore 452001 \\ ${ }^{4}$ Department of Computer Applications, S.D. Bansal College of Technology, Indore 452001 \\ e-mail: sukusap@yahoo.com, nsapre@sssits.ac.in
}

MS received 11 April 2007; revised 30 October 2007

\begin{abstract}
Lipophilicity or hydrophobicity is a crucial physico-chemical property of an oral drug compound. In the present study, we have analysed the structural parameters responsible for enhancing the lipophilicity expressed in terms of Octanol-Water partition coefficient, $\log P$, of 2-amino-6-arylsulfonylbenzonitrile (AASBN) derivatives used as NNRTIs in AIDS therapy. Connectivity based Randic $(\chi)$ and Balaban $(J)$ and atomistic Kier-Hall electrotopological state ( $E$-state) indices have been used to develop Quantitative Structure-Property Relationship (QSPR) and to predict the effect of substitution on the $\log P$. Model has been developed using multiple linear regression analysis (MLR) for the training set (67 compounds) and the model was tested on a test set ( 7 compounds). Significant results were obtained for the training set $\left(R^{2}=0.948, R_{\text {adj }}^{2}=0.939, S E=0 \cdot 177, F\right.$-ratio $\left.=101.22\right)$. The results of the test set too implicated a good fit $\left(R^{2}=0.941, R_{\text {adj }}^{2}=0.929, S E=0.157, F\right.$-ratio $\left.=80.05\right)$. Among the two connectivity based topological indices; Randic $(\chi)$ index showed better predictive ability than the Balaban $(J)$ index. Kier-Hall $E$-state indices indicated that among the functional groups, methyl, bromo, chloro groups on ring $\mathrm{A}$, with their positive coefficients enhanced the lipophilicity. Amino, cyano group on ring $\mathrm{B}$ and the bridging $\mathrm{S}, \mathrm{SO}, \mathrm{SO}_{2}$ with their negative coefficients showed an adverse effect on the lipophilicity parameter. Thus, Kier-Hall $E$-state indices along with topological indices could be well applied for deriving QSPR models and analysing substitution effects of various functional groups. The training set, correlation matrix and observed and experimental $\log P$ values are available as supplementary material for this article.
\end{abstract}

Keywords. QSPR; AASBN; NNRTIs; randic; Balaban; Kier-Hall E-state indices; MLR.

\section{Introduction}

Acquired immunodeficiency syndrome (AIDS) is a pandemic disease, whose etiologic agent is human immunodeficiency virus type-1 (HIV-1). The causative agent of AIDS, infects the cells of the immune system leading to destruction of the host immunity, generally the CD-4 helper T-cells. There are three types of viral enzymes related to HIV namely: HIVprotease, HIV-integrase and HIV-reverse transcriptase. ${ }^{1-3}$ The reverse transcriptase enzyme is an important target for the development of selective inhibitors. The HIV reverse transcriptase inhibitors are of two types: nucleoside reverse transcriptase inhibitors

\footnotetext{
*For correspondence
}

(NRTIs) and non nucleoside reverse transcriptase inhibitors (NNRTIs). The safety, selectivity and high potency of NNRTIs have made them more important in the field of drug research as compared to NRTIs. ${ }^{4}$

Structurally diverse NNRTIs such as hydroxyethoxyphenylthiothymine (HEPT) ${ }^{5}$ qinoxaline derivatives (efavirenz), ${ }^{6} \alpha$-anilinophenylacetamide ( $\alpha$-APA) derivatives, ${ }^{7} 2^{\prime}, 5^{\prime}$-bis(O-(tert-butyldimethylsilyl)-3'spiro-5"-(4"-amino-1",2"-oxathiole-2",2"-dioxide) pyrimidine (TSAO) derivatives, ${ }^{8}$ bis(heteroaryl)piperazine derivatives (BHAP) (Delavirdine), ${ }^{9}$ dipyridodiazepinone (Nevirapine), ${ }^{10}$ tetrahydroimidazobenzodiazepinone (TIBO), ${ }^{11}$ phenylethylthioureathiazole (PETT) ${ }^{12}$ have been reported in literature and out of these Nevirapine, Delavirdine and Efavirenz have been approved for the treatment of HIV-1 infection. 
The NRTIs act at the catalytic site of HIV-RT by terminating the DNA synthesis ${ }^{13}$ while NNRTIs inhibit the enzyme non-competitively to a site adjacent to deoxyribonucleoside triphosphate binding site of the enzyme. ${ }^{14-16}$ This adjacent site is approximately $10 \AA$ away from the catalytic site. The rapid development of drug resistance and mutation occurs, thus reducing the effectivity of NNRTIs to a large extent. ${ }^{17}$ Also, it is well known that the side chain and the backbone of the residues surrounding the active site pocket, adjust to each bound drug in a common fashion. Thus, this viral protein is able to accommodate inhibitors of different chemical structures.

NNRTIs of a new ring system containing AASBN derivatives have been found to effectively inhibit the replication of a variety of HIV-1 strains at the reverse transcriptase step. ${ }^{18}$ Therefore, the present work has been performed with the aims of deriving the QSPR and understanding the effects of substitution of various functional groups of these AASBN derivatives with the help of topological indices and electro-topological indices on their lipophilicity. These relationships are mathematical models that enable the prediction of properties and/or activities from the structural parameters. It is worthwhile to mention that the graphs here consist of one and the same cycle; they differ in the acyclic part only.

Mathematical encoding of the molecules according to their structural features has been used here. Transformation of a chemical structure into a mathematical graph makes it possible to express the chemical structure of these compounds by a single number and can be achieved in different ways. ${ }^{19-22}$

It appears that among many topological indices ${ }^{23-36}$ that have been proposed, the Randic connectivity index $(\chi)^{31}$ introduced by Randic and the Balaban index $(J)^{37}$ introduced by Balaban, are the most widely used indices in QSAR as well as QSPR studies. An $E$-state index is a structure descriptor for an atom within a covalently bonded molecule. The advantage of $E$-state indices is that they can be used directly as a single number molecular descriptor in QSAR/QSPR studies.

The present study has been performed with the aim of obtaining information about the structural characteristics responsible for enhancing the octanolwater partition coefficient $(\log P)$ of this class of compounds i.e. to understand the effect of substitution on their lipophilicity. Also the study is aimed at determining QSPR of HIV-1 NNRTIs for the class of AASBN derivatives. ${ }^{18}$

\section{Materials and methods}

In the present work, 74 compounds of 2-amino-6arylsulfonylbenzonitriles ${ }^{18}$ are taken and Randic, ${ }^{31}$ Balaban $^{37}$ indices (topological indices) and for understanding the substitution effect electro-topological indices are correlated with lipophilicity $\log P$. Connectivity based Randic and Balaban indices were calculated using in-house built program. $E$-state indices for various functional groups were calculated using e-calc. ${ }^{45}$ Multiple linear regression analysis was performed using systat regression software ver. 11.0. ${ }^{44}$ Firstly, a correlation matrix was derived from the program and then regression analyses were performed.

\subsection{Methodology}

2.1a Topological indices used: Two connectivity based topological indices namely, Randic connectivity index $(\chi)^{31}$ and Balaban index $(J)^{37}$ are well presented in literature. Therefore they will be described here rather briefly.

The Randic connectivity index $(\chi) \cdot{ }^{31-33}$ The connectivity index, $\chi=\chi(G)$ of $G$ is defined as

$$
{ }^{1} \chi_{R}=\sum\left[\delta_{i} \delta_{j}\right]^{-0.5}
$$

where $\delta i$ and $\delta j$ are the valencies of vertices $i$ and $j$, equal to the number of bonds connected to the atoms $i$ and $j$ in $G$, representing the graph of a compound.

The Balaban index $(J)^{37}$. The Balaban index $J=J(G)$ of $G$ is defined as:

$$
J=\left[b /(\mu+1) \sum_{i-k}\left(\delta_{i} \delta_{k}\right)^{-1 / 2},\right.
$$

where $b$ is number of bonds in $G, \mu$ is the cyclomatic number of $G$ and $\delta_{i}$ and $\delta_{k}(i$ or $k=1,2,3, N$; is the number of vertices in $G$ ) are the distance sums. The cytomatic number $\mu=\mu(G)$ of a polycyclic graph $G$ is equal to the minimum number of edges necessary to be erased from $G$ in order to transform it into the related to acyclic subgraph. In the case of monocyclic graph $\mu=1$.

2.1b Kier-Hall electrotopological state (E-state) indices $^{38-43}$ : A molecule is represented with a hydro- 
gen-suppressed graph (i.e. a chemical graph) in which the atoms are identified as elements with certain valence states. The sigma bonds are represented by dimensionless connection between the atoms. The ingredients in the chemical graph are: (a) presence of an atom (b) valance state of an atom and (c) degree of adjacency. From these ingredients, we may create a parameter reflecting the electronic and topological state of an atom in the chemical graph. Quantitatively, the topological attribute may be accomplished by using the degree of adjacency, which is equal to the count of sigma electrons contributed by an atom in the chemical graph known as $\delta$. To give primary atom the largest value of degree of adjacency, it is convenient to reciprocate the $\delta$ value (i.e. $1 / \delta$ ). The electronic attribute of an atom in a chemical graph must also be encoded into an index with the help of information about the number of pi, sigma and lone-pair of electrons associated with each atom. In addition to these, attribute related to the interactions taking place between atoms within a molecule is interpreted in terms of electronegativity of an atom. The difference between the number of valence electrons and the number of sigma electrons in an atom is known as Kier-Hall electronegativity. The valence electron count on an atom in a chemical graph is designated by $\delta^{\prime}$, which is equal to the count of valence electrons contributed by an atom in a molecule $\left(Z^{v}\right)$ minus the count of hydrogen atoms on that atom $(H)$, thus $Z^{v}-H=\delta^{v}$. Kier-Hall electronegativity is given by $\delta^{\nu}-\delta$, where, $\delta=$ sigma electrons, $\delta^{v}=$ sigma + pi + lone-pair electrons and $\delta^{\nu}-\delta=$ pi + lone pair electron.

This expression provides a way of enumerating the relative structure of an atom in a chemical graph and renders information regarding the potential of both intramolecular as well as intermolecular phenomena.

Intrinsic state of an atom in a chemical graph is shown by the formula:

$$
I=\frac{\delta^{v}+1}{\delta}
$$

The intrinsic state of an atom in a chemical graph reflects its electronic and topological attributes in the absence of interaction with rest of the molecule.

The $E$-state of an atom is explicitly given by the sum of its $I$-state value and the values of all the perturbing terms due to the remaining atoms in the molecule.
Mathematically $E$-state for the $i$ th atom is given by the expression

$$
S_{i}=I_{i}+\sum_{j} \Delta I_{i j},
$$

where the summation is over all the other atoms in a molecule.

Sum of the molecule's $E$-state values is equal to the sum of its $I$-state values.

$$
\sum_{i} S_{i}=\sum_{i} I_{i}
$$

Hence the sum depends only on the number and the type of atoms in a molecule and not on their mutual interactions.

\section{Results and discussion}

All the 67 chemical structures, taken as the training set, of 2-amino-6-arylsulfonylbenzonitrile derivatives are illustrated (see supplementary material 1). It also records the value of graph theoretical descriptors $(J, \chi)$ and Kier-Hall electrotopological state indices $\left(E_{\mathrm{NH}_{2}}, E_{\mathrm{S}}, E_{\mathrm{SO}}, E_{\mathrm{SO}_{2}}, E_{\mathrm{CH}_{3}}, E_{\mathrm{OCH}_{3}}, E_{\mathrm{TCN}}, E_{\mathrm{Cl}}\right.$, $\left.E_{\mathrm{Br}}\right)$ of AASBN analogs. The respective octanolwater partition coefficient, $\log P$ values, the position of the substituents $\left(R\right.$ and $\left.R^{\prime}\right)$ on the benzene ring where $R^{\prime}$ represents the $\mathrm{S} / \mathrm{SO} / \mathrm{SO}_{2}$ group bridging the two aromatic rings and $\mathrm{R}$ represents various substituents attached to the benzene ring $\mathrm{A}$, are also recorded in it.

The correlation matrix for the correlation of $\log P$ with the earlier mentioned structural descriptors (topological indices) and Electrotopological descriptors of AASBN derivatives are shown (see supplementary material 2).

A perusal of the correlation matrix showed that in the case of univariate correlation, among the topological indices, Balaban index $(J)$ showed better correlation potential as compared to Randic index $(\chi)$. But among the $E$-state indices $E_{\mathrm{S}}$ showed best correlation potential while $E_{\mathrm{CH}_{3}}$ showed poorest correlation potential. The $E_{\mathrm{CN}}$ and $E_{\mathrm{Br}}$ were capable of predicting the $\log P$ value to similar extent, though $E_{\mathrm{CN}}$ gave slightly better result than $E_{\mathrm{Br}}$. The order of correlation for the value of $E$-state indices and topological indices from univariate correlation matrix showed the following order:

$$
\begin{gathered}
E_{\mathrm{S}}>J>E_{\mathrm{SO}_{2}}>E_{\mathrm{SO}}>E_{\mathrm{Cl}}>\chi> \\
E_{\mathrm{CN}}>E_{\mathrm{Br}}>E_{\mathrm{NH}_{2}}>E_{\mathrm{CH}_{3}} .
\end{gathered}
$$


A QSPR model was generated using the aforementioned parameters for understanding the effect of substitution as well as the connectivity and following equation was obtained:

$$
\begin{aligned}
\log P= & 0.370( \pm 0.047) \chi-1.898 \\
& ( \pm 0.353) J-2.234( \pm 0.423) \mathrm{E}_{\mathrm{NH}_{2}}- \\
& 0.505( \pm 0.364) E_{\mathrm{S}}-0.202( \pm 0.045) E_{\mathrm{SO}}- \\
& 0.109( \pm 0.022) E_{\mathrm{SO}_{2}}-0.830( \pm 0.220) E_{\mathrm{TCN}}+ \\
& 0.319( \pm 0.034) E_{\mathrm{CH}_{3}}+0.132( \pm 0.010) E_{\mathrm{Cl}}+ \\
& 0.303( \pm 0.021) E_{\mathrm{Br}}+26.422
\end{aligned}
$$

$n=66, R=0.974, R^{2}=0.948, R_{\text {adj }}^{2}=0.939, \mathrm{SE}=0.177$, $F$-ratio $=101 \cdot 22$, outlier $=$ Comp. 65 .

The quality of the model was considered as statistically satisfactory on the basis of the above mentioned results. In the aforementioned equation, connectivity based Randic index showed a high positive coefficient where as Balaban index showed a high negative coefficient. But in the case of $E$ state indices a high positive coefficient for $E_{\mathrm{CH}_{3}}$ indicates that the presence of $\mathrm{CH}_{3}$ group will have an enhancing effect on the $\log P$ value. Among the halo substitutents, high positive coefficient for $\mathrm{Br}$ as compared to $\mathrm{Cl}$ shows that, a bulky and less elcetronegative halogen substitutent will have an enhancing effect on the lipophilicity. A high negative coefficient for $E_{\mathrm{NH}_{2}}$ and $E_{\mathrm{CN}}$ indicates that the presence of nucleophilic groups such as $\mathrm{NH}_{2}$ and $\mathrm{CN}$ on ring $\mathrm{B}$ will adversely affect the octanol-water partition coefficient. As far as bridging groups are concerned, the negative coefficients of $E_{\mathrm{S}}, E_{\mathrm{SO}}$ and $E_{\mathrm{SO}_{2}}$ in decreasing order indicates that they all have an unfavourable impact on the $\log P$ values and also suggests that there should be a more bulky bridging group than $\mathrm{SO}_{2}$ to have a better lipophilicity.

The $\log P$ values for the AASBN derivatives have been calculated using the best multivariate correlation. Such calculated $\log P$ values are recorded (see supplementary material 3 ). The observed (experimental) $\log P$ values are also given. The quality of correlations has been demonstrated by their residual values, i.e. the difference between the observed and the calculated $\log P$ values. The residuals obtained from the best correlation are also given therein.

A graph showing correlation of observed and calculated $\log P$ values for training set is presented in figure 1 .

A test set of 7 compounds as given in table 1 has been taken to verify the validity of model obtained from aforementioned calculations.

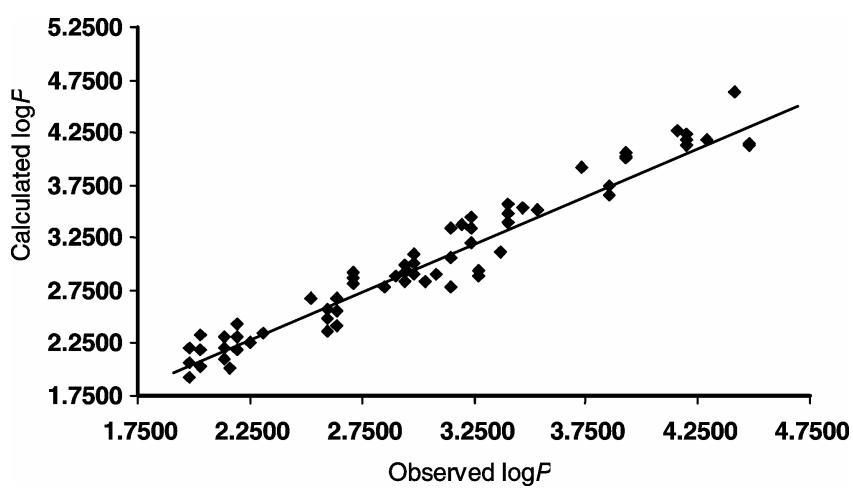

Figure 1. A plot of observed and predicted $\log P$ values for AASBN derivatives for training set.

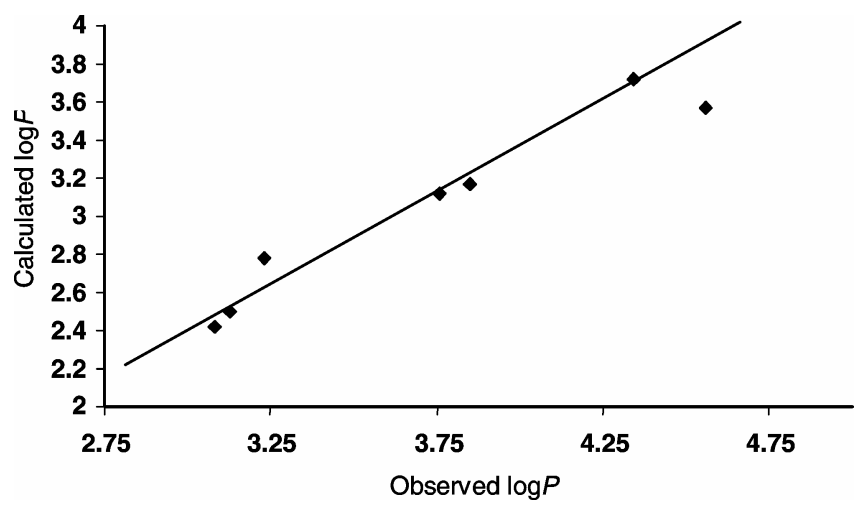

Figure 2. A plot of observed and predicted $\log P$ values for AASBN derivatives for test set.

Table 2 presents the observed and calculated $\log P$ values for the test set. The regression thus obtained for the observed and calculated $\log P$ values shows a high degree of relatedness.

A graph showing correlation of observed and calculated $\log P$ values for test set is presented in figure 2 .

The above results of test set are self indicative of fitness of correlation in predicting the connectivity as well as substitution effect in AASBN derivatives.

\section{Conclusions}

The present study related to 2-amino-6-arylsulfonylbenzonitrile derivatives leads us to make the following conclusions:

The results have indicated that among the two topological indices; Randic $(\chi)$ index showed a better predictive capability as compared to the Balaban $(J)$ index. Among the functional groups with positive coefficient of $E$-state values on ring $\mathrm{A} ; \mathrm{CH}_{3}$ group showed the highest positive value, while among the 
Table 1. Octanol/water partition coefficient $(\log P)$, topological indices $(\chi, J)$ and $E$-state indices $\left(E_{\mathrm{NH}_{2}}, E_{\mathrm{S}}, E_{\mathrm{SO}}\right.$, $\left.E_{\mathrm{SO}_{2}}, E_{\mathrm{CH}_{3}}, E_{\mathrm{OCH}_{3}}, E_{\mathrm{TCN}}, E_{\mathrm{Cl}}, E_{\mathrm{Br}}\right)$ of AASBN analogues (test set).

\begin{tabular}{|c|c|c|c|c|c|c|c|c|c|c|c|c|}
\hline$R$ & $R^{\prime}$ & $\log P$ & $\chi$ & $J$ & $E_{\mathrm{NH}_{2}}$ & $E_{\mathrm{S}}$ & $E_{\mathrm{SO}}$ & $E_{\mathrm{SO}_{2}}$ & $E_{\mathrm{TCN}}$ & $E_{\mathrm{CH}_{3}}$ & $E_{\mathrm{CL}}$ & $E_{\mathrm{Br}}$ \\
\hline $4-\mathrm{CH}_{3}$ & $\mathrm{~S}$ & $3 \cdot 850$ & $9 \cdot 113$ & 1.907 & 5.774 & 1.559 & $0 \cdot 000$ & $0 \cdot 000$ & $11 \cdot 228$ & $0 \cdot 000$ & 0.000 & $0 \cdot 0$ \\
\hline $3-\mathrm{NH}_{2}$ & $\mathrm{~S}$ & $4 \cdot 56$ & $10 \cdot 024$ & 1.934 & 5.755 & $1 \cdot 485$ & $0 \cdot 000$ & $0 \cdot 000$ & $11 \cdot 185$ & $0 \cdot 000$ & 0.000 & $0 \cdot 0$ \\
\hline $3,5-(\mathrm{CH} 3)_{2}$ & S & $4 \cdot 340$ & $9 \cdot 507$ & 1.957 & $5 \cdot 809$ & $1 \cdot 579$ & $0 \cdot 000$ & $0 \cdot 000$ & $11 \cdot 311$ & $2 \cdot 071$ & $0 \cdot 000$ & $0 \cdot 0$ \\
\hline $3,5-(\mathrm{CH} 3)_{2}$ & SO & $3 \cdot 080$ & $9 \cdot 935$ & $2 \cdot 067$ & 5.751 & $0 \cdot 000$ & $11 \cdot 195$ & $0 \cdot 000$ & $11 \cdot 165$ & 1.957 & $0 \cdot 000$ & $0 \cdot 0$ \\
\hline $2,5-\mathrm{Cl}_{2}$ & $\mathrm{SO}$ & $3 \cdot 230$ & $9 \cdot 952$ & $2 \cdot 098$ & 5.700 & $0 \cdot 000$ & $10 \cdot 933$ & $0 \cdot 000$ & 11.051 & $0 \cdot 000$ & 5.832 & $0 \cdot 0$ \\
\hline $3,5-(\mathrm{CH} 3)_{2}$ & $\mathrm{SO}_{2}$ & $3 \cdot 130$ & $10 \cdot 268$ & $2 \cdot 183$ & 5.679 & $0 \cdot 000$ & $0 \cdot 000$ & $21 \cdot 575$ & $10 \cdot 989$ & $1 \cdot 830$ & $0 \cdot 000$ & $0 \cdot 0$ \\
\hline $3-\mathrm{O}(\mathrm{CH} 2)_{3} \mathrm{CH}_{3}, 5-\mathrm{CH}_{3}$ & $\mathrm{SO}_{2}$ & $3 \cdot 760$ & $12 \cdot 306$ & $2 \cdot 100$ & 5.736 & 0.000 & 0.000 & $22 \cdot 014$ & $11 \cdot 118$ & $1 \cdot 802$ & 0.000 & $0 \cdot 0$ \\
\hline
\end{tabular}

Table 2. Calculated and observed $\log P$ values of 2amino-6-arylsulfonylbenzonitriles analogues (test set) derived from the regression (7).

\begin{tabular}{lcc}
\hline $\log P$ (obsd.) & $\log P$ (calc.) & $\begin{array}{c}\text { Residual }=\log P(\text { obsd. })- \\
\log P(\text { calc. })\end{array}$ \\
\hline 3.850 & $3 \cdot 170$ & 0.6800 \\
4.560 & 3.569 & 0.9910 \\
4.340 & 3.723 & 0.6170 \\
3.080 & 2.423 & 0.6570 \\
3.230 & 2.777 & 0.4530 \\
3.130 & 2.501 & 0.6290 \\
3.760 & 3.122 & 0.6380 \\
\hline
\end{tabular}

$\log P($ calc. $)=1 \cdot 145( \pm 0 \cdot 128) \log P($ obs. $)+0 \cdot 224$

$\left(n=7, R=0.970, R^{2}=0.941, R_{\mathrm{adj}}^{2}=0.929, \mathrm{SE}=0.157\right.$, $F$-ratio $=80 \cdot 05)$

halo substituents, $\mathrm{Br}$ seemed to be better as compared to $\mathrm{Cl}$ indicating that, a bulky and a less electronegative halogen group would enhance the lipophilicity. The functional groups with negative coefficient were $\mathrm{NH}_{2}$ and $\mathrm{CN}$ (on ring $\mathrm{B}$ ) indicating that they would have an adverse effect on compound's lipophilicity, suggesting that, the presence of electrophilic groups on ring B may help in enhancing the magnitude of octanol/water partition coefficient. The E-state values for the bridging atoms also showed different negative coefficients in the order, $\mathrm{S}>\mathrm{SO}>$ $\mathrm{SO}_{2}$. This indicates that there should be a more bulky group than $\mathrm{SO}_{2}$ in the referred position, which would help in enhancing the $\log P$ value. The test set too showed a good linearity between the observed and the calculated $\log P$ values, thus validating the aforementioned inferences.

Thus, the results suggest that the methyl and bulky halogens can play a major role in deciding the lipophilic character of these compounds. Also, new and more potent compounds with enhanced lipophilicity could be designed and synthesized, utilizing the above information regarding electrophilicity of a group attached to it. On the basis of above results, these structure based indices ascertain their applicability in choosing the substituents in a chemical entity, so as to obtain a potent and novel molecule.

\section{Acknowledgements}

The authors thank Dr R C Sarswat, Director, and Dr P K Sen, Dean (R\&D), SGSITS. Indore, for providing computing facilities.

\section{References}

1. Kubinyi H 1994 Quant. Struct.-Act. Relat. 13285

2. De Clercq E 1996 Rev. Med. Virol. 697

3. De Clercq E 1999 Farmaco 5426

4. Campiani G, Ramunno A, Maga G, Nacci V, Fattorusso C, Catalanotti B, Morelli E and Novellino E 2002 Curr. Pharm. Des. 8615

5. Baba M, Tanakas H, De Clercq E, Pauwels R, Balzarini J, Schols D, Nakashima $\mathrm{H}$, Perno C F, Walker R T and Miyasaka T 1989 Biochem. Biophys. Res. Commun. 1651375

6. Kleim J P, Bender R, Billhardt U M, Meichsner C, Riess G, Rosner M, Winkler I and Paessens A 1993 Antimicrobial. Agents Chemotherapy 371659

7. Pauwels R, Andries K, Debyser Z, Van Daele P, Schols D, Vandamme A M, Janssen C G M, Anne J, Cauwenbergh G, Desmyter J, Heykants J, Janssen M A C, De Clercq E and Janssen P A J 1993 Proc. Natl. Acad. Sci. (USA) 901711

8. Balzarini J, Pérez-Pérez M J, San-Felix A, Schols, Perno D C F, Vandamme A, Camarasa M J and De Clercq E 1992 Proc. Natl. Acad. Sci. (USA) 89 4392

9. Romero D L, Busso M, Tan C K, Reusser F, Palmer J R, Poppe S M, Aristoff P F, Downey K M, So A G, Resnick L and Tarpley W G 1991 Proc. Nat. Acad. Sci. 888806

10. Merluzzi V J, Hargrave K D, Labadia M, Grozinger K, Skoog M, Wu J C, Shih C K, Eckner K, Hattox S and Adams J 1990 Science 2501411 
11. Pauwels R, Andries K, Desmyter J, Schols D, Kukla M J, Breslin H J, Raeymaeckers A, Van Gelder J, Woestenborghs R and Heykants J 1990 Nature (London) 343470

12. Ahgren C, Backro K, Bell F W, Cantrell A S, Clemens M, Colacino J M, Deeter J B, Engelhardt J A, Hogberg M, Jaskunas S R and Johanssons N G 1995 Antimicrobial Agents and Chemotherapy 391329

13. To date six nucleosides have been approved by the FDA: AZT, 3TC, d4T, ddI, ddC, and abacavir

14. Mager P P 1997 Med. Res. Rev. 17235

15. Arnold E, Das K, Ding J, Yadav P N, Hsiou Y, Boyer P L and Hughes S H 1996 Drug Des. Discov. 1329

16. Jonckheere H, Anne J and De Clercq E 2000 Med. Res. Rev. 20129

17. Richman D, Shih C K, Lowy I, Rose J, Prodanovich P, Goff S and Griffin 1991 J. Proc. Natl. Acad. Sci. (USA) 8811241

18. Chan Joseph H $2001 \mathrm{~J}$. Med. Chem. 441866

19. Lucic B, Nikolic S, Trinajstic N, Juric A and Mihalic Z 1995 Croat. Chem. Acta 68417

20. Randic M and Seybold P G 1993 SAR-QSAR Environ. Res. 177

21. Randic M 1992 J. Math. Chem. 997

22. Trinajstic N 1983 Chemical graph theory (Florida: CRC Press) vol 2, pp 3-70

23. Seybold P G, May M and Bagal U A $1987 \mathrm{~J}$. Chem. Educ. 64575

24. Gutman I and Polansky P E 1989 Mathematical concepts in organic chemistry (Berlin: Springer-Verlag) pp 23-120

25. King R B 1987 Chemical applications of topology and graph theory (Amsterdam: Elsevier) pp 1-47
26. Merrifield R E and Simmons H E 1989 Topological methods in chemistry (New York: Wiley) pp 67-90

27. Trinajstic N, Sabijic A and Nikolic S 1986 Acta. Pharm. Jugosel. 361

28. King R B and Rouvray D H 1987 Graph theory and topology in chemistry (Amsterdam: Elsevier) pp 1-50

29. Trinajstic N, Nicolic S and Carter S 1989 QSAR: Theory Appl. 38469

30. Randic M 1975 J. Am. Chem. Soc. 976609

31. Randic M 1991 J. Chem. Inf. Comput. Sci. 31311

32. Randic M 1991 New J. Chem. 18517

33. Mihalic Z, Nikolic S, Trinajstic N 1992 J. Chem. Inf. Comput. Sci. 3228

34. Bonchev D 1983 Information theoretic indices for characterization of chemical structures (Chichester: Research Studies Press) pp 23-57

35. Mandloi M et al $2000 \mathrm{~J}$. Chem. Inf. Comput. Sci. 40 57

36. Dobrynin Gutman I 1994 Pib. De L'inst. Math. 5618

37. Balaban A T 1982 Chem. Phys. Lett. 80399

38. Kier L B and Hall L H 1999 Molecular structure description: The electro-topological state (San Diego: Academic Press) pp 20-58

39. Kier L B and Hall L H 1993 J. Chem. Inf. Comput. Sci. 33598

40. Kier L B, Hall L H and Mohney B K 1991 Quant. Structure-Activity Relationships 1043

41. Testa B and Kier L B 1991 Med. Res. Rev. 1135

42. Kier L B and Hall L H 1994 Quant. StructureActivity Relationships $\mathbf{1 2} 383$

43. Kier L B and Hall L H 1997 Med. Chem. Res. 7335

44. SYSTAT 10.0 SYSTAT Software Inc. USA

45. Kier-Hall, E-calc version 1.1, 1999 